Kodjo Attikpoé

\title{
La langue de la littérature pour la jeunesse : une lecture des Confidences de Médor de Micheline Coulibaly et des Cendres du père de Pius Ngandu Nkashama
}

Résumé: En raison de la capacité de lecture limitée de son public cible, la littérature de jeunesse adopte souvent le principe de simplicité, considéré comme sa principale caractéristique. Cette simplicité se manifeste syntaxiquement et lexicalement dans le récit. Loin d'être dévalorisante, elle constitue une valeur esthétique en soi.

Cependant, la critique littéraire a montré que la littérature jeunesse contemporaine tend vers la complexité. Cet article vise à analyser cette complexité du point de vue de la langue dans la littérature destinée aux jeunes lecteurs en Afrique francophone subsaharienne. Pour ce faire, nous examinons deux romans : d'une part, Les Confidences de Médor de l'Ivoirienne Micheline Coulibaly, qui recourt à un langage humoristique, et d'autre part, Les Cendres du père de l'écrivain congolais Pius Ngandu Nkashama, qui traite du thème de la violence, et dans lequel l'arsenal lexical utilisé par l'auteur contribue à l'atmosphère sombre du roman.

Mots clés : Afrique, roman pour la jeunesse, langue, simplicité, complexité, humour, violence.

\section{Observations introductives}

La critique n'a pas fini de souligner les ambiguïtés de la littérature pour la jeunesse, le « grand livre des paradoxes » (Prince 2012, 9). L'une d'elles tient à sa « dénomination problématique » (Nières-Chevrel 2009, 13). Par exemple, dans les années 1970, l'un des grands débats consistait à savoir laquelle des formules « littérature de jeunesse » et « littérature pour la jeunesse » rend mieux compte du rapport entre littérature et lectorat juvénile (Nières-Chevrel 2009, 16-17). En effet, à la différence de la préposition « de », le « pour » ${ }^{1}$ indique d'une manière plus explicite « l'inten-

1 Selon Isabelle Nières-Chevrel, beaucoup de chercheurs avaient opté, à l'époque, pour la dénomination « littérature de jeunesse ». Ce débat est loin d'être clos. Bertrand Ferrier, pour sa part, récuse la formule « littérature de jeunesse » : «Ce livre s’intéresse à la littérature pour la jeunesse, donc ni à la "littérature jeunesse" (la jeunesse n’est pas un adjectif), qui évite le "pour” comme

Ә Open Access. (C) 2021 Kodjo Attikpoé, published by De Gruyter. (c) BY-NC-ND This work is licensed under the Creative Commons Attribution-NonCommercial-NoDerivatives 4.0 International License. 
tionnalité constitutive de l'œuvre » (Prud'homme 2007, 22). Cependant, cette intentionnalité, voire «surintentionnalité » (Prud'homme 2007, 22), est aussi équivoque dans la mesure où la notion de jeune lecteur - le destinataire spécifique -, censée tracer une ligne de partage entre littérature pour la jeunesse et celle destinée aux adultes, apparaît d'autant plus ambivalente qu'elle donne lieu à des jugements dépréciatifs : parler d'une littérature destinée à un public spécifique, dont la capacité à décoder les signes linguistiques semble restreinte, impliquerait de produire une littérature qui n'est pas à même de répondre aux exigences esthétiques de la littérature avec un grand « L », d'où les préjugés qui ravalent très souvent la littérature pour la jeunesse au rang de littérature secondaire.

Pourtant, celle-ci se veut aussi œuvre de création et revendique la littérarité au sens jakobsonien : « ce qui fait d'une œuvre donnée une œuvre littéraire » (Jakobson 1977, 16). Ici, Mikhaïl Bakhtine a montré le traitement esthétique et artistique subi par le langage dans le roman (Bakhtine 1978). Aussi ce dernier est-il « conçu comme phénomène de la vie littéraire-artistique et non de la vie quotidienne » (Bakhtine 1984, 267). S’agissant de la prose romanesque pour la jeunesse, il importe que la critique étudie davantage les articulations entre langue et fiction. Johanne Prud'homme notait que l'utilisation du « registre soutenu privilégié à un usage familier de la langue » a partie liée avec l'aspect pédagogique indissociable de l'intentionnalité immanente de l'œuvre pour la jeunesse (Prud'homme 2005, 22). Toutefois, à notre sens, cette question de la langue devrait être appréhendée d'un autre point de vue : la langue de l'écrivain pour la jeunesse donne-t-elle à voir une certaine poéticité ? Quels sont « les aperçus langagiers énoncés par les textes eux-mêmes » ? (Gauvin 2004, 12). S’intéresser aussi au « bruissement de la langue » (Barthes 1984) perceptible dans la fiction romanesque pour la jeunesse permet de rendre justice à cette littérature. Est-il besoin de rappeler qu'à cause de leur utilitarisme, mais aussi de leur perception comme production « simple », « rarement, en effet, les livres pour la jeunesse sont valorisés pour leur créativité [...] [langagière] » (Ferrier 2009, 253) ?

Même si, de nos jours, la critique a montré que « les écrivains pour la jeunesse contemporains mettent en question les conventions, les codes et les normes qui ont régi traditionnellement le genre » en utilisant des « techniques narratives complexes (polyfocalisation, discours métafictif, mélange de genres, refus de clôture, intertextualité, ironie, parodie) » (Beckett 2003, 73), il n’en demeure pas

si elle avait honte de définir un horizon de réception spécifique à cette production, ni à la "littérature de jeunesse" : la littérature pour la jeunesse n'est ni une littérature exclusivement sur la jeunesse, ni une littérature datant de la jeunesse de l'auteur, ce qui permettrait de parler d'une œuvre littéraire de jeunesse » (Ferrier 2009, 15). 
moins que la simplicité passe pour la caractéristique principale de la littérature pour la jeunesse.

Jusqu'ici, les théories et discours critiques de cette littérature ont été élaborés essentiellement à partir des œuvres du « centre », notamment des pays occidentaux, qui disposent d'une longue tradition littéraire. Cependant, on assiste aussi de nos jours à l'essor d'une littérature pour la jeunesse dans les pays de la "périphérie », dont ceux d'Afrique francophone. L'étude de ces « nouvelles littératures » permettra aussi de mettre en lumière des éléments susceptibles d'enrichir le discours critique.

L'objectif du présent article est d'examiner les stratégies langagières dans deux romans d'Afrique francophone : Les Confidences de Médor (1996) de l'Ivoirienne Micheline Coulibaly et Les Cendres du père ${ }^{2}$ (2014) du Congolais Pius Ngandu Nkashama. Ces deux auteurs ont en commun d'enjamber les frontières de la littérature : la première a fait son entrée sur la scène littéraire en écrivant d'abord pour les enfants, et ensuite pour les adultes. Le second, lui, fera le chemin inverse. Les deux romans ici à l'étude diffèrent par leur tonalité : Les Confidences de Médor construit une poétique du rire à travers un récit plein d'humour, tandis que Les Cendres du père, comme le titre le suggère, met en place une écriture de la violence. Quelles formes prennent les médiations langagières dans ces romans ? Le fait de circuler aux frontières de la littérature influe-t-il sur les stratégies scripturales et langagières des auteurs ? A-t-on ici affaire à une composition simple ou plutôt complexe ? Le roman africain pour la jeunesse reflète-t-il aussi la " surconscience linguistique de l'écrivain francophone »? (Gauvin 1997, 5). Avant d'aborder l'analyse des œuvres, nous donnerons un bref aperçu de la question liée à la simplicité comme trait majeur de la littérature pour la jeunesse.

\section{Simplicité versus complexité ?}

Nonobstant son caractère ambivalent, la notion de simplicité passe souvent pour une valeur esthétique et un principe d'écriture. Un coup d'œil dans le dictionnaire révèle quelques exemples : «Qualité du style simple » (Littré) ; «Qualité de ce qui est simple »: Simplicité de style. Grande simplicité d'expression. La simplicité d'un plan, d'une composition, d'une méthode. (Dictionnaire de l'Académie française) ; « la qualité d'une chose sans éléments superflus (1579), à propos de la langue, en particulier dans le domaine esthétique (1669) » (Dictionnaire historique de

2 Le roman Les Cendres du père a été initialement publié sous le titre Le Fils du mercenaire en 1995 aux Éditions NEA/EDICEF. 
la langue française, Le Robert). Dans La nostalgie du simple. Essai sur les représentations de la simplicité dans le discours critique au XIX $X^{e}$ siècle, Mariane Bury a montré comment « la réflexion sur la simplicité se trouve au cœur des débats esthétiques » (Bury 2004, 79), et elle ajoute que « tout au long du siècle, la simplicité représente un idéal formel », qui renvoie à « l'idée de grandeur et de perfection, de l'homme ainsi que de l'écriture » (Bury 2004, 229).

Cependant, cette simplicité devient suspecte lorsqu'elle opère dans le champ de la littérature pour la jeunesse. Dans son essai Einfachheit als Kategorie der Kinderliteratur (La simplicité comme catégorie de la littérature pour la jeunesse) paru en 1984, la critique allemande Maria Lypp interroge la notion de simplicité, et tente de déconstruire les perceptions négatives qui entourent la littérature pour la jeunesse comme étant une production peu exigeante. Elle part du constat selon lequel la simplicité sous-jacente au livre destiné au jeune lecteur répond principalement au souci de tenir compte des compétences de lectures supposées restreintes du destinataire ; et de là découlent les jugements qui minimisent la littérarité de l'œuvre pour la jeunesse. Mais selon Maria Lypp, la réalité est toute autre : la prose fictionnelle pour la jeunesse, pour être pleinement réceptionnée par ce jeune lecteur, exige justement la mise en place de mécanismes complexes. En outre, si l'on considère, par exemple, les nombreux problèmes liés à la réception des enfants et les diverses voies à emprunter pour les résoudre, il apparaît tout à fait problématique de qualifier de simple toute la littérature pour la jeunesse. Bref, la simplicité, comprise comme caractère distinctif ou trait servant à délimiter les frontières de la littérature de jeunesse par rapport à la littérature générale, s'avérera inadéquate tant qu'elle revêt un caractère réducteur (Lypp 1984, 9).

Pour notre part, il faut ajouter que si la notion de simplicité semble dévalorisante en littérature pour la jeunesse, c'est aussi parce que celle-ci donne lieu à une production abondante où nombre de livres sont dépourvus d'ambitions esthétiques. « Tout n'est pas littérature » en effet, pour emprunter cette formule au titre d'un essai de Bertrand Ferrier (2009). Paul Hazard abonde dans le même sens en dénonçant « des livres faux et indigestes » (Hazard 1967, 60). Ainsi, il prône l'esthétisation de l'œuvre pour la jeunesse, fait l'éloge de la simplicité, en mettant en avant les livres qui « restent fidèles à l'essence même de l'art, c'est-àdire qui proposent aux enfants un mode de connaissance intuitif et direct, une beauté simple capable d'être immédiatement perçue » (Hazard 1967, 57). Il cite en exemple Charles Perrault : «Perrault est frais comme une aube ; on ne finit pas de découvrir ses qualités. De la malice, de l'humour. [...] Et ce clair langage ! Et cette simplicité, "la seule qualité qu'on ne se lasse jamais de louer" comme dit SainteBeuve, la seule qui touche toutes les âmes... » (Hazard 1967, 20).

Dans la même veine, nombre de critiques s'évertuent à démontrer les vertus de la simplicité, cherchant ainsi à gommer l'éternel cliché de sous-littérature qui 
colle à la littérature pour la jeunesse. C’est ainsi que Nathalie Prince affirme : « Le corpus de la littérature de jeunesse permet finalement d'illustrer ce travail paradoxal de sophistication par la simplification qui, quand il est abouti, ne coïncide pas avec un affaiblissement de la qualité littéraire du texte mais au contraire multiplie l'espace interprétatif et intensifie le champ des possibles. » (Prince 2013).

Dans Tout n'est pas littérature ! La littérarité à l'épreuve des romans pour la jeunesse, Bertrand Ferrier, pour sa part, s'attache à mettre en lumière les marqueurs de littérarité du roman pour la jeunesse. Il se penche, entre autres, sur les aspects linguistiques et syntaxiques. Selon lui, une littérarité spécifique à la littérature de jeunesse n'implique pas de rabaisser l'enfant : « [N]on, s’adresser à la jeunesse ne suppose pas d'utiliser un vocabulaire nécessairement pauvre ; [...] cela ne suppose pas non plus [...] de produire un discours au niveau de langue systématiquement respectable et suffisamment neutre [...] » (Ferrier 2009, 255-256). De même, « la simplicité du vocabulaire n'est donc pas ennemie d'une certaine richesse littéraire ! (Ferrier 2009, 159). Qu'en-est-il donc de notre corpus ?

\section{Les Confidences de Médor : une poétique du rire}

Micheline Coulibaly, l'une des pionnières de la littérature de jeunesse en Côte d'Ivoire, signe en 1996 Les Confidences de Médor, dont la force réside dans la mise en place d'une poétique du comique. La quatrième de couverture affiche, d'entrée de jeu, l'intention esthétique du roman, qui consiste à utiliser l'humour comme principe d'écriture : « Micheline Coulibaly [...] propose un récit plein d'humour aux adolescents ». Cette tonalité du récit est surtout liée à la nature et au statut du narrateur : un chien qui prend plaisir à porter son regard sur ses maîtres, et par ricochet, sur la société : « À travers cette vision “canine” de l'espèce humaine, Micheline Coulibaly nous invite à rire de nos travers » (quatrième de couverture).

Ce court roman adopte une langue plutôt classique, " soutenue », riche et très précise par endroits. Le lexique d'environ 35 mots proposé à la fin du roman témoigne non seulement de la richesse du vocabulaire, mais aussi du désir de l'auteur de ne pas rabaisser le lecteur juvénile. Prenons par exemple l'incipit qui contient un mot expliqué dans ce lexique non exhaustif ${ }^{3}$ : « Les margouillats se poursuivent inlassablement le long des murs de la clôture » (3) ; "D’une manière

3 Les expressions et les mots suivants auraient pu aussi figurer dans le lexique du roman : manège (12), Mégère (13), tourments (31), boudinée (32), « je ne m’en formalise pas » (34), hargneux (36), fulgurante (37), lorgner avec méfiance (46), enamourée (61), se morfondre (61). 
inlassable, sans se fatiguer, sans s'arrêter » (69). Autrement dit, si l'auteure avait été guidée par le souci de proposer au jeune lecteur un vocabulaire "simple », elle aurait tout simplement remplacé dans le texte « inlassablement par «sans se fatiguer » ou par « sans s’arrêter ».

Au-delà de la richesse du langage et de l'acquisition du vocabulaire, l'enjeu primordial de notre analyse est de mettre en évidence les articulations entre le langage et les effets comiques qui structurent l'univers fictionnel du roman. Dans Le rire. Essai sur la signification du comique, Henri Bergson distingue entre le comique de mots et le comique de situation, mais souligne que «le comique de mots suit de près le comique de situation » (Bergson 1958, 99). Si les effets comiques se produisent aussi très souvent par l'intermédiaire du langage, l'auteur fait observer cependant qu'il « faut distinguer entre le comique que le langage exprime, et celui que le langage crée » (Bergson 1958, 79).

Dans Les Confidences de Médor, l'anthropomorphisme constitue un élément essentiel de cette poétique du rire : l'observation de la société à travers le regard d'un chien, l'un des plus fidèles compagnons de l'homme, contribue à susciter aussi le comique de situation : " Il n'y a pas de comique en dehors de ce qui est proprement humain. [...] On rira d'un animal, mais parce qu'on aura surpris chez lui une attitude d'homme ou une expression humaine » (Bergson 1958, 2-3). Médor, le chien narrateur, adopte, dès l'incipit, un ton ironique en évoquant sa « nouvelle condition sociale » qui lui « interdit certaines choses » (3) et lui vaut certains ennuis. Pour le lecteur familier du discours ambiant dans certaines sociétés africaines, la phrase « Je ne suis pas n’importe qui ! » (3), mise ici dans la bouche du narrateur canin, a une saveur ironique dans la mesure où elle fait allusion à l'orgueil et à la vanité des gens qui se croient importants. De même, le chien se moque non seulement de son propre nom, « Médor », qui témoigne du « manque d'imagination » de ses maîtres, mais aussi de celui de ces derniers : ils « s’appellent les Pokassé. Entre nous, je préfère encore m’appeler Médor ! » (3). Le lecteur est tenté de voir dans le nom de ces maîtres une sorte de jeu de mot ironique, homophone de « pot cassé ». Par ailleurs, l'anthropomorphisme du chien sert également à railler le complexe de supériorité des humains et leur propension à vouloir ériger des frontières entre les hommes, en somme des « classes sociales », comme on le constate dans l'épisode où le narrateur raconte sa vie et celle de sa mère dénommée Patience. Quand celle-ci, « une chienne de si basse extraction $^{4} »(14)$, est séduite par Tonnerre, un berger allemand, le maître de ce dernier s'emporte :

4 La recherche du registre soutenu a sans doute amené l'auteure à utiliser ce terme vieilli. 
Quelle idée, Tonnerre! Toi avec cette chienne pleine de puces ? gronda Monsieur Duroc.

- Ici, Tonnerre! Ne t’approche plus de cette chienne. Nous t’avons déjà choisi une fiancée.

Princesse est plus digne de toi car elle a un pedigree aussi impressionnant que le tien. (14)

Ici, le mot «pedigree » peut être compris aussi dans un sens ironique. Le lexique à la fin du livre l'explique simplement par « origine familiale ». Mais une lecture plus attentive permet d'affirmer qu'il se réfère à la fois au sens premier relatif au monde animal et au second sens (ironique), selon Le Petit Robert : " 1. Extrait du livre généalogique d'un animal de race pure. Établir le pedigree d'un chien de luxe. 2. (Personnes). Iron. Généalogie (il est fier de son pedigree et de sa particule) ». Cette rencontre entre deux chiens de pedigrees opposés sert également prétexte à l'auteur pour porter un regard critique sur la notion de race dans le discours des humains :

- Alors, vous êtes vraiment d'origine allemande ? demanda Patience en baissant pudiquement les yeux?

- Auriez-vous des préjugés raciaux, jeune fille ? s’inquiéta Tonnerre (13).

Médor pointe du doigt cette attitude discriminatoire des hommes avec un humour qui n'échappe pas au lecteur :

Notre mère était donc une magnifique chienne de race indéterminée, selon le jargon des humains qui ont la manie de vouloir classer les êtres et les choses. Est-ce si important ? Il faut plutôt s'en tenir à la valeur de l'individu et non se préoccuper de sa race ou de son origine. Enfin race indéterminée ou pas, aux dires des chiens qui l'ont connue dans sa jeunesse, Patience était une beauté (8).

Le terme « jargon » semble ici aussi péjoratif, même si l'explication que donne le lexique du roman passe sous silence cet aspect : «Langage particulier à un groupe et qui contient des mots créés par ce groupe » (69).

Le narrateur canin tourne en dérision l'arrivisme de ses maîtres, qui affichent désormais un nouveau style de vie. Étant donné que certains quartiers urbains confèrent un statut social, l'arrivée au sommet de " la hiérarchie sociale » des Pokassé va d'abord se traduire par un changement de lieu : « D’Adjamé [quartier populaire], nous sommes donc "montés” à la Riviera, le quartier résidentiel par excellence » (24). Monter est mis entre guillemets, ce qui peut vouloir dire qu'il s'agit d'un terme familier ou qu'aux yeux de Médor, cette ascension sociale et géographique de ses maîtres n'est que factice. Dans ce sens, il faut remarquer que le narrateur, qui utilise le langage soutenu, prend soin de mettre entre guillemets des termes familiers tels que "être vacciné contre ", " craquer » pour un homme. Mais s'agissant du mot « affaires », Médor semble douter de l'honnêteté de son maître : « Depuis, nous avons considérablement évolué car Karim Pokassé 
s'est lancé avec succès dans les "affaires". C’est un mot bien vague pour désigner toutes les combines auxquelles se livrent ceux qui cherchent à s'enrichir aux dépens des autres » (24). Pour les Pokassé, l'ascension sociale doit se traduire aussi par des signes extérieurs. Avec beaucoup d'humour, le chien décrit leurs nouvelles habitudes alimentaires, qui les poussent à engager un chef cuisinier " sixième catégorie », qui leur cuisine de la " nourriture raffinée » occidentale, en somme, des "menus sophistiqués fort peu adaptés aux palais de mes chers patrons » (25). Il va particulièrement railler le changement physique de Mariama Pokassé, qui se blanchit la peau. La dépigmentation volontaire de la peau, phénomène qui semble prendre de l'ampleur chez certaines Africaines, traduit un déni ou une haine de soi. Le rire a " une signification et une portée spéciales », (Bergson 1958, 101) et « par la crainte qu'il inspire, il réprime les excentricités, [...], assouplit enfin tout ce qui peut rester de raideur mécanique à la surface du corps social » (Bergson 1948, 15). Ainsi, Médor raille l'excentricité que constitue le désir de changer de couleur de peau. Dans L'Ironie littéraire. Essai sur les formes de l'écriture oblique, Philippe Hamon note :

Mais le texte littéraire se communique par l'écriture et en différé. [...] Aucun corps, aucune figure (visage) ne sont présents dans l'univers du livre imprimé. [...] L'écrivain ironique devra donc trouver des moyens de compenser cette "perte », et utiliser tous les registres de la langue pour signaler au lecteur inconnu ses intentions. (Hamon 1996, 79-80)

Pour décrire le visage transformé de madame Pokassé, le chien narrateur va recourir à la symbolique des couleurs et à deux types d'énoncés. La période de normalité, c'est-à-dire antérieure à celle de la transformation excentrique, est soulignée par une phrase brève : «Autrefois, son regard simplement souligné de Khôl me chavirait » (28) ; en revanche, le changement de style suscité par le désir de ressembler aux femmes qu'elle voit "dans les magazines et à la télévision » nécessite un énoncé plus détaillé, qui consiste à faire un gros plan sur le nouveau visage « bigarré » de la patronne :

Maintenant, Mariama affiche un visage chargé, comme une palette de peintre : les sourcils dessinés en traits marron foncé, les paupières peintes en violet, les cils raidis au mascara vert, les pommettes rehaussées d'un rose bonbon et la bouche écarlate. Qui dit mieux ? Est-ce cela la beauté africaine ? Comment lui faire comprendre qu'elle n'a pas besoin de tous ces artifices pour nous conquérir, Karim et moi ? (28)

Pour montrer que Mariama Pokassé n'est pas le seul personnage " excentrique », Médor représente également sous un jour moqueur et humoristique madame Blofoè - amie de sa maîtresse -, devenue elle aussi une " créature bigarrée » (32) : «Elle avait complètement changé de teint, passant du beau noir d'ébène que je lui connaissais à un jaune inégal lui donnant un air maladif » (31). Ce ton railleur, 
à travers lequel le narrateur invite la femme noire à s’accepter soi-même, devient plus incisif, lorsque ce dernier tente de faire une comparaison avec la condition canine : « J'espère qu'elle a compris que l'essentiel, c'est de se sentir bien dans sa peau. Ne voit-elle pas comment nous autres, chiens, nous sommes bien dans la nôtre? Chaque race a son pelage et chacun est heureux avec le sien. Je m'imagine mal en train de désirer les boucles d'un caniche ou les oreilles d'un cocker » (29).

Cette écriture jubilatoire, qui met en avant le rire comme une « espèce de brimade sociale » (Bergson 1958, 103), contraste ici avec l'atmosphère de l'univers romanesque ancré dans la représentation des guerres historiques et d'autres formes de conflits.

\section{Les Cendres du père : une écriture de la violence}

La violence est une thématique omniprésente dans la littérature africaine ( $\mathrm{Ba} 2012$; Bidima et Zoungbo 2016 ; Munzenza 2017). Le récit africain pour la jeunesse, lui aussi, s'inscrit dans cette dynamique de « conjuration du mal par l'écrit » (Ngandu Nkashama 2011, 25), en mettant en place une esthétique de dénonciation de la violence moderne, qui affecte particulièrement l'enfance africaine (Attikpoé 2008).

Le thème de la violence est au cœur de l'œuvre de l'auteur congolais Pius Ngandu Nkashama (voir Plaiche 2017), qu'il s'agisse des romans pour adultes ou de sa prose destinée à la jeunesse. Déjà, on peut noter que le titre de certaines de ces œuvres suggère l'idée de violence, telle que la guerre, la mort ${ }^{5}$, comme c'est aussi le cas du roman pour la jeunesse Les Cendres du père. Le titre Le Fils $d u$ mercenaire, sous lequel ce roman a été initialement publié, fait plus explicitement référence à une guerre historique, notamment celle menée par le Congo pour se libérer du joug colonial. Dans le roman, Yannick, vivant à Bruxelles, part au Congo à la recherche de son père ayant combattu comme mercenaire durant cette guerre. Ce voyage va donner lieu à la remémoration de cette guerre, mais fournira aussi à l'auteur l'occasion de mettre en scène les violences politiques et sociales sous le régime du dictateur défunt Mobutu qui, néanmoins, n’est pas nommé dans le récit.

La complexité du dispositif langagier et narratif du roman donne à penser qu'on est en présence de ce que la critique anglo-saxonne appelle « crossover fiction » (Beckett 1999, 2009), c'est-à-dire une prose qui se prête à un double lec-

5 S’agissant des romans pour adultes, on peut citer : La Malédiction (1983); La Mort faite homme (1986); Les Étoiles écrasées (1988). 
torat d'adolescents et d'adultes. Ce qui frappe à la lecture du roman, c'est surtout l'abondance des descriptions complexes : « Un matin interminable, encombré de miasmes et de clameurs bruyantes qui s’agglutinaient pour saturer la mémoire aussi abruptes que les forces surgies de la terre. Un sanglot ébranlait la brousse enfoncée dans une coquille de pierre dure, au pied de la montagne » (38). Par ailleurs, dans tous les aspects du récit, l'écriture de l'auteur semble être travaillée par l'obsession de suggérer, à travers le choix des mots, l'idée de combat, de colère, de turbulence, de frayeur, de nervosité, d'excitation, etc., même dans des épisodes qui n’ont pas de lien direct avec les violences politiques, le thème central du roman. Par exemple, dans la phrase « Ils fixaient le soleil intense qui montait haut avant d'assiéger le ciel » (49), le verbe « assiéger » sert à caractériser certes le déplacement du soleil, mais dénote aussi la violence, dans la mesure où il s'inscrit dans le champ lexical de la guerre. De même, le jeune protagoniste Yannick, bien qu'obsédé par la quête du père, se trouve ébranlé par des sentiments que le narrateur décrit en des termes qui évoquent également l'atmosphère de combat : « Le jour du départ, Yannick avait connu des songes troublés par d'affreuses ${ }^{6}$ hallucinations. Il aurait fendu la nuit à coups de griffes, chaque fois qu'il pouvait se délivrer des monstres qui ne lui ramenaient que des obsessions » (32). Son arrivée à l'aéroport entraîne des descriptions s'appuyant sur le champ lexical de la colère : « Les haut-parleurs hurlaient des messages hachés (33). Plus loin : «Les écrans sonores aboyèrent un départ imminent » (34). Au Congo, l'indigence des salles de l'aéroport est également décrite avec un vocabulaire évoquant implicitement le sang :

Les rumeurs des voix se mélangeaient aux frottements des pieds sur le sol fendillé. Les carrelages déteints et ébréchés risquaient d'arracher les peaux nues des talons imprudents [...] c'était cela aussi, la terre natale. (37)

Dans cette écriture que nous qualifierons de mouvementée, la nature est aussi perçue comme source de violence :

Enfin, le jour du départ s'était éclairé mais n'avait exhibé qu'un soleil moribond. Bruxelles endurait un ciel gris, tellement bas qu'il oppressait la poitrine. Les silhouettes des passantes circulaient tête baissée, comme si elles allaient buter contre les fantômes. (30)

Même si c'est la période hivernale qui est mise en scène ici, il n'en demeure pas moins que les termes comme " moribond » et « fantômes » font penser, dans un autre champ sémantique, à l'idée de la mort. En Afrique, le soleil va être dépeint sous sa face terrifiante. Qu'il se montre le matin ou au zénith, ses effets sont d'une

6 Désormais, certains mots seront mis en italique dans les citations par nos soins. 
violence cruelle : « Le matin se levait et une chaleur étouffante démangeait la peau. Elle brûlait comme du piment sur une blessure fraîche qui entame la chair » (35) ; plus loin : «Quand le soleil monte au zénith, l'atelier chauffe à l'extrême, un véritable brasier. Les corps rôtissent comme sur la braise » (47). Dans ces deux exemples, on le voit, le narrateur utilise la comparaison pour peindre l'image des souffrances atroces infligées par le soleil. Si le premier exemple évoque la souffrance et la torture, le deuxième, lui, exprime davantage l'idée de la mort par le feu. En somme, toutes les conditions atmosphériques sont marquées du sceau de la violence :

La pluie s'est mise à vitupérer. Le ciel déversait des trombes d'eau en des cascades puissantes. Les arbres gémissaient. Des craquements lugubres s'entendaient au loin. Le vent impétueux les fouettait. Des lamentations comme des feulements rauques. [...] Yannick s'allongea sur le même grabat que Grand-Mère. [...] Désormais, il savait qu'il était destiné à braver la violence de la guerre dévastatrice. (101)

Dans cette citation, la description des effets de la nature s'achève sur l'évocation de la « violence de la guerre dévastatrice ». Le narrateur établit un lien entre les violences « atmosphériques » et celles des hommes.

Dans le roman, la mémoire de la guerre est ressuscitée surtout à travers le personnage du colonel Schoenen, un mercenaire. Il raconte cette guerre à Yannick, et « prétendait avoir mené des batailles glorieuses sous le commandement du père de Yannick » (20-21). Il souligne particulièrement la férocité de ce dernier, surnommé « Kifiakyo. Celui qui nettoie tout, qui tue lors de son passage et ne laisse même pas de miettes » (22). Aussi utilise-t-il l'hyperbole pour décrire l'atrocité de cette guerre historique : «[P]uis nous les avons décimés. Le matin, toute la rivière était rougie du sang des corps criblés de balles que se disputaient les hyènes » (21).

La représentation de la mémoire de cette guerre se double de la mise en scène des bestialités commises sous le régime d’oppression en contexte postcolonial au Congo. En arrivant sur sa terre natale, Yannick sera aussitôt témoin de la « rage bestiale » (69) des soldats mutins qui n'épargnent même pas les enfants : « Les enfants sortirent en silence. Ils assistaient, impuissants, au spectacle d'anéantissement du fruit de leur travail » (69). Comme dans son roman pour la jeunesse Un matin pour Loubène $e^{7}$, l'auteur utilise le lexique de l'animalité pour caractériser le

7 Le roman Un Matin pour Loubène (1991) de Pius Ngandu Nkashama décrit aussi la brutalisation de l'enfant Loubène par les forces de Sécurité. Ici, le narrateur utilise le lexique animalier : « Il le regarde s'avancer comme un animal féroce. Il se dit que cet homme va se transformer en un bouledogue fruste et cruel, avec une gueule bleu noir et des crocs pointus [...]. Le monstre émet des grognements plaintifs et fonce sur le garçon » (38); ou encore : "La bête-féroce de tout à l'heure se fige en une posture idiote. [...] L'homme-bête murmure entre ses dents » (40). 
comportement des militaires : «Des camions militaires bondés passaient au loin et leur tuyauteries lançaient des mugissements de mastodontes. Les soldats arboraient des treillis aux couleurs agressives. Certains montraient des dents féroces et brandissaient des mitraillettes contre les enfants. [...] L’armée avait toujours représenté l'épouvante » (44). Ici, les voix du narrateur et de l'enfant se rejoignent au sujet de la cruauté bestiale des militaires : Madiya, l'un des enfants débrouillards avec qui Yannick s'est lié d'amitié à son arrivée, tient un discours sur son pays en s’appuyant sur des faits qui pourraient sembler invraisemblables au lecteur non averti : «Les brigades présidentielles se sont illustrées ces derniers temps. La nuit, sans se faire annoncer, les éclaireurs ont jailli en véritables commandos. [...] Ils ont égorgé une centaine d'étudiants [...] un carnage ${ }^{8}$ (44). L'auteur se sert ainsi de l'enfant comme porte-voix pour déployer un langage rabaissant le dictateur : « Le Président, un petit minable sans scrupules. Pitoyable chauffeur de taxi de son état. Une petite frappe venue de l'étranger. Là-bas, il vendait des œufs pourris le long des artères des bidonvilles » (45). C'est dire que le président-dictateur, premier responsable de toutes les violences cruelles dans son pays, ne mérite aucun respect. Malgré son caractère violent, ce discours offensant apparaît tout à fait légitime.

\section{Conclusion}

Notre analyse des romans Les Confidences de Médor et Les Cendres du Père corrobore la tendance selon laquelle la fiction contemporaine destinée aux jeunes lecteurs déploie de plus en plus des procédés et des techniques narratives complexes. En réalité, cette complexité n'est pas antinomique avec la simplicité comme valeur esthétique, fruit d'un processus tout aussi complexe. Elle est simplement plus manifeste, et a cependant l'avantage d'articuler de manière plus souveraine la littérarité du livre pour la jeunesse, ainsi que ses rapports dynamiques avec la littérature générale.

Au-delà des catégories de complexité, de simplicité et de normativité, nous avons cherché à mettre en lumière les modes d'esthétisation qui s'opère par les médiations langagières. Le roman Les Confidences de Médor recourt à des éléments de la transposition tels que l'humour et l'ironie (Bergson 1958, 94) comme procédé artistique pour affirmer sa littérarité. En thématisant les guerres histo-

8 Le lecteur familier de l'histoire du Congo reconnaît ici un fait réel qui s’est produit au début des années 1990. 
riques et les violences politiques en contexte postcolonial, Les Cendres du père déploie une écriture où la charge négative des mots renforce considérablement l'atmosphère noire de la narration.

\section{Bibliographie}

Attikpoé, Kodjo. « L'empreinte de la violence dans le roman de jeunesse en Afrique francophone ». L'inscription du social dans le roman pour la jeunesse. Kodjo Attikpoé (dir.). Paris : L'Harmattan, 2008. 181-202.

$\mathrm{Ba}$, Mamadou Kalidou. Nouvelles tendances du roman africain francophone contemporain (1990-2010). De la narration de la violence à la violence narrative. Paris : L'Harmattan, 2012.

Bakhtine, Mikhaïl. Esthétique et théorie du roman. Paris: Gallimard, 1978.

Bakhtine, Mikhaïl. Esthétique de la création verbale. Paris : Gallimard, 1984.

Barthes, Roland. Le bruissement de la langue. Paris : Seuil, 1984.

Beckett, Sandra L. «Roman pour tous ? » Perspectives contemporaines du roman pour la jeunesse. Virginie Douglas (dir.). Paris : L'Harmattan, 2003. 57-73.

Beckett, Sandra L. Crossover Fiction. New York et Londres : Routledge, 2009.

Beckett, Sandra L. (dir.). Transcending Boundaries: Writing for a Dual Audience of Children and Adults. New York et Londres : Garland, 1999.

Bergson, Henri. Le rire. Essai sur la signification du comique. Paris : Presses Universitaires de France, 1958.

Bidima, Jean Godefroy, et Victorien Lavou Zoungbo (dir.). Réalités et représentations de la violence en postcolonies. Perpignan : Presses universitaires de Perpignan, 2016.

Bury, Mariane. La nostalgie du simple : essai sur les représentations de la simplicité dans le discours critique au XIXe siècle. Paris : Champion, 2004.

Coulibaly, Micheline. Les Confidences de Médor. Abidjan : Edilis 1996.

Ferrier, Bertrand. Tout n'est pas littérature ! La littérarité à l'épreuve des romans pour la jeunesse, préface de Marie-Aude Murail. Rennes : Presses universitaires de Rennes, 2009.

Gauvin, Lise. L'écrivain francophone à la croisée des langues. Entretiens. Paris : Karthala, 1997.

Hamon, Philippe. L'ironie littéraire : essai sur les formes de l'écriture oblique. Paris : Hachette Supérieur, 1996.

Hazard, Paul. Les livres, les enfants et les hommes. Paris : Hatier, 1967.

Jakobson, Roman. Huit questions de poétique. Paris: Seuil, 1977.

Lypp, Maria. Einfachheit als Kategorie der Kinderliteratur. Francfort-sur-le-Main : dipa-Verlag, 1984.

Munzenza, Willy Kangulumba. Une esthétique dans le cri : écriture de la violence et violence de l'écriture dans le roman congolais de la fin du XX' siècle. Paris : L'Harmattan, 2017.

Ngandu Nkashama, Pius. Un matin pour Loubène. Ville LaSalle (Québec) : Hurtubise, 1991.

Ngandu Nkashama, Pius. Le Fils du mercenaire. Paris et Dakar : NEA/EDICEF, 1995.

Ngandu Nkashama, Pius. Guerres africaines et écritures historiques. Paris : L'Harmattan, 2011.

Ngandu Nkashama, Pius. Les Cendres du père. Paris : L'Harmattan, 2014.

Nières-Chevrel, Isabelle. Introduction à la littérature de jeunesse. Paris : Didier jeunesse, 2009. 
Plaiche, Karel. « Littérature et tragédies historiques : penser les écritures de guerre à l'ère contemporaine. (Entretien avec Pius Ngandu Nkashama) ». Tracks and Traces of Violence: Representation and Memorialization of Violence. Views from Art, Literature and Anthropology. BIGSAS-Workgroup (dir.). Münster : LIT, 2017. 117-134.

Prince, Nathalie. La littérature de jeunesse : pour une théorie littéraire. Paris : Armand Colin, 2012.

Prince, Nathalie. «Préambule à la simplicité ». La simplicité, une notion complexe ?. Nathalie Prince et Patricia Eichel-Lojkine (dir.). Publije 3 (2013). http://publije.univ-lemans.fr/ publije/index.php?id=168. Le Mans : Le Mans Université (20 octobre 2017).

Prud'homme, Johanne. "Entre nation et mondialisation : Questions fondamentales sur la nature de la littérature pour la jeunesse ». Nationalités, mondialisation et littératures et de jeunesse. Bernard Huber et Guy Missodey (dir.). Paris : Éditions des archives contemporaines, 2007. 19-28.

Kodjo Attikpoé is a professor in the Department of Modern Languages, Literatures and Cultures at Memorial University of Newfoundland, Canada. He is the author of numerous publications, including: Linscription du social dans le roman contemporain pour la jeunesse, (ed., 2008), L'image de l'enfant dans les conflits (co-editor, with Jean Foucault, 2013), Poétique de l'enfance : perspectives contemporaines (ed., 2017), and Les pouvoirs de la littérature de jeunesse (ed., 2018). 